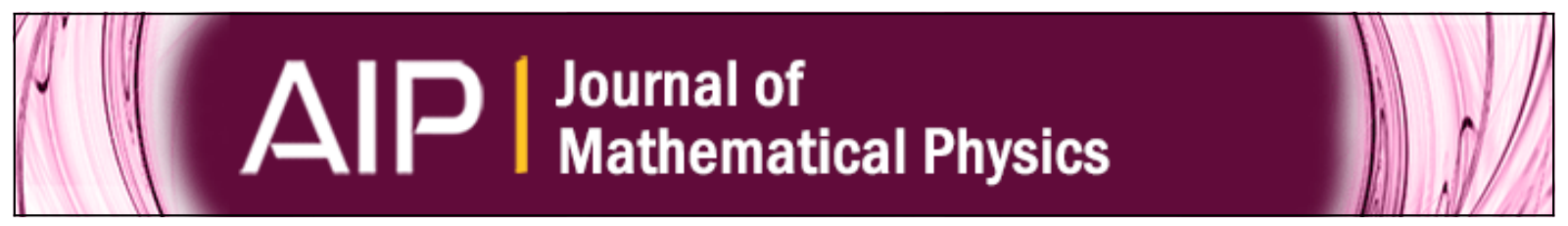

\title{
Integrable quasiclassical deformations of cubic curves
}

Y. Kodama, B. Konopelchenko, L. Martínez Alonso, and E. Medina

Citation: Journal of Mathematical Physics 46, 113502 (2005); doi: 10.1063/1.2101067

View online: http://dx.doi.org/10.1063/1.2101067

View Table of Contents: http://scitation.aip.org/content/aip/journal/jmp/46/11?ver=pdfcov

Published by the AIP Publishing

\section{Articles you may be interested in}

Deformations of loop algebras and integrable systems: hierarchies of integrable equations

J. Math. Phys. 45, 4578 (2004); 10.1063/1.1804229

Deformations of the Monge/Riemann hierarchy and approximately integrable systems

J. Math. Phys. 44, 251 (2003); 10.1063/1.1522134

Deformations of surfaces associated with integrable Gauss-Mainardi-Codazzi equations

J. Math. Phys. 41, 2251 (2000); 10.1063/1.533237

Response to "Comment on 'Generalized W $\infty$ symmetry algebra of the conditionally integrable nonlinear evolution equation'” [J. Math. Phys. 40, 3685 (1999)]

J. Math. Phys. 40, 3691 (1999); 10.1063/1.532917

Comment on "Generalized W $\infty$ symmetry algebra of the conditionally integrable nonlinear evolution equation" [J. Math. Phys. 36, $3492(1995)]$

J. Math. Phys. 40, 3685 (1999); 10.1063/1.532916

\section{Did your publisher get}

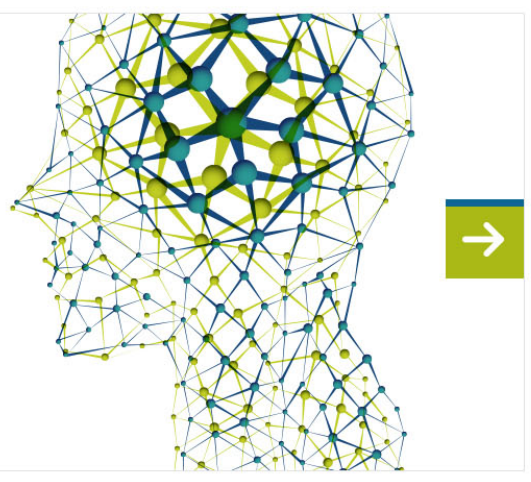




\section{Integrable quasiclassical deformations of cubic curves}

\section{Y. Kodama}

Department of Mathematics, Ohio State University, Columbus, Ohio 43210

B. Konopelchenko

Dipartimento di Fisica, Universitá di Lecce and Sezione INFN, 73100 Lecce, Italy

L. Martínez Alonso

Departamento de Física Teórica II, Universidad Complutense, E28040 Madrid, Spain

E. Medina

Departamento de Matemáticas, Universidad de Cádiz, E11510 Puerto Real, Cádiz, Spain

(Received 18 February 2005; accepted 6 September 2005; published online 9 November 2005)

A general scheme for determining and studying hydrodynamic type systems describing integrable deformations of algebraic curves is applied to cubic curves. Lagrange resolvents of the theory of cubic equations are used to derive and characterize these deformations. (C) 2005 American Institute of Physics.

[DOI: $10.1063 / 1.2101067]$

\section{INTRODUCTION}

The theory of algebraic curves is a fundamental ingredient in the analysis of integrable nonlinear differential equations as it is shown, for example, by its relevance in the description of the finite-gap solutions or the formulation of the Whitham averaging method. ${ }^{1-8}$ A particularly interesting problem is characterizing and classifying integrable deformations of algebraic curves. Indeed, as it is shown in a series of recent papers, ${ }^{9-12}$ these deformations lie at the crossroads of intricate connections between the theory of random matrices and several models of Laplacian growth processes. It turns out that the integrable models underlying these applications are members of the Whitham hierarchies of dispersionless integrable models introduced by Krichever in Refs. 6 and 7. Moreover, the corresponding solutions are usually characterized in terms of solutions of hydrodynamic systems.

Natural deformations of algebraic curves arise in the dynamics of the algebraic orbits ${ }^{7}$ of the Whitham hierarchies. For example, algebraic orbits of the Zabolotskaya-Khokhlov dispersionless KP (dKP) hierarchy

$$
\frac{\partial k}{\partial t_{n}}=\left\{Q_{n}, k\right\}, \quad n \geqslant 1,
$$

where

$$
k=p+\sum_{n=1}^{\infty} \frac{a_{n}(x, t)}{p^{n}}, \quad Q_{n}:=\left(k^{n}\right)_{\geqslant 0}, \quad t:=\left(t_{1}, t_{2}, \ldots\right)
$$

are deformations of algebraic curves

$$
f(k)=E(p, x, t),
$$

where $E=E(p, x, t)$ is a meromorphic function of $p$, such that (3) determines a reduction of the dKP hierarchy. In the Gelfand-Dikii case the function $E$ depends on the variables $(x, t)$ through a finite number of functions which evolve according to a system of hydrodynamic type. Neverthe- 
less, other deformations of algebraic curves have been formulated which do not correspond to algebraic orbits of the standard dispersionless hierarchies. For example, the integrable hierarchy associated to the energy-dependent Schrödinger problem ${ }^{13}$ admits a dispersionless limit which leads to a family of deformations of the curves ${ }^{14,15}$

$$
p^{2}=k^{2 N+1}+\sum_{n=1}^{N} u_{n} k^{2 n-1} .
$$

These curves do not constitute any reduction of the dKP hierarchy and, as it is shown in Ref. 14, their deformations must be formulated in terms of the singular sectors of a dKdV Grassmannian structure.

A different approach for determining integrable deformations of general algebraic curves $\mathcal{C}$ defined by monic polynomial equations,

$$
\mathcal{C}: F(p, k):=p^{N}-\sum_{n=1}^{N} u_{n}(k) p^{N-n}=0, \quad u_{n} \in \mathbb{C}[k],
$$

was proposed in Refs. 14 and 15. It applies for finding deformations $\mathcal{C}(x, t)$ of $\mathcal{C}$ with the deformation parameters $(x, t)$, such that the multiple-valued function $\mathbf{p}=\mathbf{p}(k)$ determined by (5) obeys an equation of the form of conservation laws

$$
\partial_{t} \mathbf{p}=\partial_{x} \mathbf{Q},
$$

where the flux $\mathbf{Q}$ is given by an element from $\mathrm{C}[k, p] / \mathcal{C}$,

$$
\mathbf{Q}=\sum_{r=1}^{N} a_{r}(k, x, t) \mathbf{p}^{r-1}, \quad a_{r} \in \mathrm{C}[k] .
$$

Starting with (6), changing to the dynamical variables $u_{n}$ and using Lenard-type relations (see Ref. 15) one gets a scheme for finding consistent deformations of (5). One should also note that (6) provides an infinite number of conservation laws, when one expands $\mathbf{p}$ and $\mathbf{Q}$ in Laurent series in $z$ with $k=z^{r}$ for some $r$. In this sense, we say that Eq. (6) is integrable.

Our strategy can be applied to the generic case where the coefficients (potentials) $u_{n}$ of (5) are general polynomials in $k$,

$$
u_{n}(k)=\sum_{i=0}^{d_{n}} u_{n, i} k^{i},
$$

with all the coefficients $u_{n, i}$ being considered as independent dynamical variables, i.e., $u_{n, i}$ $=u_{n, i}(x, t)$. However, with appropriate modifications, the scheme can be also applied to cases in which constraints on the potentials are imposed. A complete description of these deformations for the generic case of hyperelliptic curves $(N=2)$ was given in Ref. 15.

The present paper is devoted to the deformations of cubic curves $(N=3)$,

$$
p^{3}-w p^{2}-v p-u=0, \quad u, v, w \in \mathrm{C}[k],
$$

and it considers not only the generic case but also the important constrained case $w \equiv 0$. Although some of the curves may be conformally equivalent (with, for example, the dispersionless Miura transformation), we will not discuss the classification problem under this equivalence in this paper (we will discuss the details of the problem elsewhere). In Sec. II a general approach to construct integrable deformations of algebraic curves is reported briefly. Section III is devoted to the analysis of the cubic case (7). We emphasize the role of Lagrange resolvents, describe the Hamiltonian structure of integrable deformations and present several illustrative examples including Whithamtype deformations. 


\section{SCHEMES OF DEFORMATIONS OF ALGEBRAIC CURVES}

In order to describe deformations of the curve $\mathcal{C}$ defined by (5), one may use the potentials $u_{n}$, as well as the $N$ branches $p_{i}=p_{i}(k)(i=1, \ldots, N)$ of the multiple-valued function $\mathbf{p}=\mathbf{p}(k)$ satisfying

$$
F(p, k)=\prod_{i=1}^{N}\left(p-p_{i}(k)\right) .
$$

The potentials can be expressed as elementary symmetric polynomials $s_{n}$ (Refs. 16-18) of the branches $p_{i}$,

$$
u_{n}=(-1)^{n-1} s_{n}\left(p_{1}, p_{2}, \ldots\right)=(-1)^{n-1} \sum_{1 \leqslant i_{1}<\cdots<i_{n} \leqslant N} p_{i_{1}} \cdots p_{i_{n}} .
$$

However, notice that, according to the famous Abel theorem, ${ }^{16}$ for $N>4$ the branches $p_{i}$ of the generic equation (5) cannot be written in terms of the potentials $u_{n}$ by means of rational operations and radicals.

There is an important result concerning the branches $p_{i}$ which is useful in our analysis. Let $\mathrm{C}((\lambda))$ denote the field of Laurent series in $\lambda$ with at most a finite number of terms with positive powers,

$$
\sum_{n=-\infty}^{N} c_{n} \lambda^{n}, \quad N \in \mathbb{Z} .
$$

Then we have the following. ${ }^{19,20}$

Theorem 1 (Newton theorem): There exists a positive integer $l$ such that the $N$ branches

$$
p_{i}(z):=\left.\left(p_{i}(k)\right)\right|_{k=z^{l},}
$$

are elements of $\mathrm{C}((z))$. Furthermore, if $F(p, k)$ is irreducible as a polynomial over the field $\mathrm{C}((k))$ then $l_{0}=N$ is the least permissible $l$ and the branches $p_{i}(z)$ can be labelled so that

$$
p_{i}(z)=p_{N}\left(\epsilon^{i} z\right), \quad \epsilon:=\exp \frac{2 \pi i}{N} .
$$

Notation convention: Henceforth, given an algebraic curve $\mathcal{C}$ we will denote by $z$ the variable associated with the least positive integer $l_{0}$ for which the substitution $k=z_{0}^{l_{0}}$ implies $p_{i}$ $\in \mathrm{C}((z)), \forall i$.The number $l_{0}$ will be referred to as the Newton exponent of the curve.

For the generic case the method proposed in Ref. 15 may be summarized as follows: Given an algebraic curve (5), we define an evolution equation for $\mathbf{u}:=\left(u_{1}, \ldots, u_{N}\right)$ in the form

$$
\partial_{t} \mathbf{u}=J_{0}\left(T \nabla_{\mathbf{u}} R\right)_{+}, \quad R(z, \mathbf{p})=\sum_{i} f_{i}(z) p_{i}
$$

where $(\cdot)_{+}$indicates the part of non-negative powers of a Laurent series in $k$ and

$$
\begin{gathered}
f_{i} \in \mathbb{C}((z)), \quad \nabla_{\mathbf{u}} R:=\left(\frac{\partial R}{\partial u_{1}} \ldots \frac{\partial R}{\partial u_{N}}\right)^{\top}, \\
J_{0}:=T^{\top} V^{\top} \partial_{x} V,
\end{gathered}
$$




$$
T:=\left(\begin{array}{cccc}
1 & -u_{1} & \cdots & -u_{N-1} \\
0 & 1 & \cdots & -u_{N-2} \\
& & \cdots & \\
0 & & \cdots & 1
\end{array}\right), \quad V:=\left(\begin{array}{cccc}
1 & p_{1} & \cdots & p_{1}^{N-1} \\
& & \cdots & \\
& & \cdots & \\
1 & p_{N} & \cdots & p_{N}^{N-1}
\end{array}\right) .
$$

Let $d_{n m}$ and $d_{n}$ be the degrees of the matrix elements $\left(J_{0}\right)_{n m}$ and the potentials $u_{n}$ as polynomials in $k$, respectively. Then (11) defines a deformation of the curve, if $d_{n m}$ and $d_{n}$ satisfy the consistency conditions

$$
\max \left\{d_{n m}, m=1,2,3\right\} \leqslant d_{n}+1, \quad n=1,2,3,
$$

and the components of $\nabla_{\mathbf{u}} R$ are in $\mathrm{C}((k))$ with $k=z^{l_{0}}$.

Equivalently, in terms of branches

$$
\mathbf{p}:=\left(p_{1}, \ldots, p_{N}\right)^{\top},
$$

the system (11) can be written as

$$
\partial_{t} \mathbf{p}=\partial_{x}\left(V \mathbf{r}_{+}\right),
$$

where

$$
\mathbf{r}:=T \nabla_{\mathbf{u}} R(z, \mathbf{p})=V^{-1} \mathbf{f}(z),
$$

with $\mathbf{f}(z):=\left(f_{1}(z), \ldots, f_{N}(z)\right)^{\top}$. Notice that $\mathbf{r}$ is a solution of the Lenard relation

$$
J_{0} \mathbf{r}=0 .
$$

Although there is not a general procedure for analyzing constrained cases, one may try a similar strategy. First, we start from the equation for branches (6) and then, by expressing the potentials in terms of the independent branches only, we look for a formulation of the flows as

$$
\partial_{t} \mathbf{u}=J_{0} \mathbf{a}, \quad \mathbf{a}:=\left(a_{1}, \ldots, a_{N}\right)^{\top},
$$

for a certain operator $J_{0}$. Finally, we use solutions $\mathbf{r}$ of Lenard relations (17) and set $\mathbf{a}=\mathbf{r}_{+}$.

Another scheme for defining integrable deformations of algebraic curves of genus zero (i.e., rational curve) is implicit in the theory of integrable systems of dispersionless type developed in Refs. 7 and 8 which we refer to them as the Whitham deformations. It is concerned with algebraic curves characterized by equations of the form

$$
k=p^{N}+v_{N-2} p^{N-2}+\cdots+v_{0}+\sum_{r=1}^{M} \sum_{i=1}^{n_{r}} \frac{v_{r, i}}{\left(p-w_{r}\right)^{i}},
$$

where $v_{n}, v_{r, i}, w_{r}$ are $k$-independent coefficients. These curves arise in the theory of algebraic orbits of the genus-zero Whitham hierarchy, ${ }^{7,8}$ where the function $k$ represents the LandauGinzburg potential of the associated topological field theory. We may rewrite the equation of the curve (19) in the polynomial form (5) with potentials $u_{n}$ of degrees $d_{n} \leqslant 1$ and satisfying a certain system of constraints.

To describe the deformations of (19) determined by Whitham flows we introduce local coordinates $\left\{z_{0}, z_{1}, \ldots, z_{M}\right\}$ of the extended $p$-plane at the punctures $\left\{w_{0}:=\infty, w_{1}, \ldots, w_{M}\right\}$ such that

$$
k=z_{0}^{N}=z_{1}^{n_{1}}=\cdots=z_{n_{M}}^{n_{M}} .
$$

It is clear that there are $N$ branches of $\mathbf{p}$ which have expansions in powers of $k^{1 / N}$ and that, for each puncture $w_{r},(r=1, \ldots, M)$, there are $n_{r}$ branches of $\mathbf{p}$ having expansions in powers of $k^{1 / n_{r}}$. Therefore, the Newton exponent $l_{0}$ is given by the least common multiple of the set of integers 
$\left\{N, n_{1}, \ldots, n_{M}\right\}$. Furthermore, it is clear that only in the absence of finite punctures $(M=0)$ the curve (19) is irreducible over $\mathrm{C}((k))$.

At each puncture in $\left\{\infty, w_{1}, \ldots, w_{M}\right\}$, there is an infinite family of Whitham deformations of (19). They can be expressed by equations of the form (see Refs. 7 and 8)

$$
\partial_{t} \mathbf{p}=\partial_{x} \mathbf{Q}_{\alpha, n},
$$

where

$$
\begin{gathered}
\mathbf{Q}_{\alpha, n}=\left(z_{\alpha}^{n}\right)_{\oplus}(\mathbf{p}), \quad \alpha=0,1, \ldots, M, n \geqslant 1, \\
\mathbf{Q}_{r, 0}=\ln \left(\mathbf{p}-w_{r}\right), \quad r=1, \ldots, M .
\end{gathered}
$$

Here $\left(z_{\alpha}^{n}\right)_{\oplus}$ stands for the singular part of $z_{\alpha}^{n}(p)$ at the puncture $w_{\alpha}$, with $\left(z_{r}^{n}\right)_{\oplus}(\infty)=0$ for $1 \leqslant r$ $\leqslant M$. There exist also commuting flows for the negative $n$ in (21) with logarithmic terms which correspond to the descendant flows of $Q_{r, 0}$ (see Ref. 8 for the details).

In the absence of finite punctures $(M=0)$, Whitham deformations become the dispersionless Gelfand-Dikii flows. They can be described by our scheme ${ }^{15}$ as the reductions $u_{1} \equiv 0, u_{N}=k-v_{0}$ of the generic case corresponding to $d_{n}=\delta_{N n}$. However, for $M \geqslant 1$ it can be seen that, in general, Whitham deformations of (19) are not reductions of the flows (11) provided by our method. Some examples of this situation for cubic curves are shown below.

\section{DEFORMATIONS OF CUBIC CURVES}

For our subsequent analysis we introduce a basic tool of the theory of third order polynomial equations, ${ }^{16}$ the so-called Lagrange resolvents, defined by

$$
\mathcal{L}_{i}:=\sum_{j=1}^{3}\left(\epsilon^{i}\right)^{j} p_{j}, \quad i=1,2,3, \quad \epsilon:=e^{2 \pi i / 3},
$$

or, equivalently,

$$
\begin{gathered}
\mathcal{L}_{1}:=\epsilon p_{1}+\epsilon^{2} p_{2}+p_{3}, \\
\mathcal{L}_{2}:=\epsilon^{2} p_{1}+\epsilon p_{2}+p_{3}, \\
\mathcal{L}_{3}:=p_{1}+p_{2}+p_{3} .
\end{gathered}
$$

They can be expressed in terms of the potentials $\mathbf{u}=(w, v, u)^{\top}$ by using the identities

$$
\begin{aligned}
& \mathcal{L}_{1} \cdot \mathcal{L}_{2}=3 v+w^{2}, \quad \mathcal{L}_{3}=w, \\
& \mathcal{L}_{1}^{3}+\mathcal{L}_{2}^{3}=27 u+9 v w+2 w^{3},
\end{aligned}
$$

which lead to

$$
\begin{aligned}
& 2 \mathcal{L}_{1}^{3}=27 u+9 v w+2 w^{3}+\sqrt{\left(27 u+9 v w+2 w^{3}\right)^{2}-4\left(3 v+w^{2}\right)^{3}}, \\
& 2 \mathcal{L}_{2}^{3}=27 u+9 v w+2 w^{3}-\sqrt{\left(27 u+9 v w+2 w^{3}\right)^{2}-4\left(3 v+w^{2}\right)^{3}} .
\end{aligned}
$$

The fundamental advantage of Lagrange resolvents is that they provide explicit expressions of the branches $p_{i}$ in terms of the potentials according to Cardano formulas 


$$
3 p_{i}=\sum_{j=1}^{3}\left(\epsilon^{-i}\right)^{j} \mathcal{L}_{j}, \quad i=1,2,3,
$$

or, equivalently,

$$
\begin{gathered}
3 p_{1}=\epsilon^{2} \mathcal{L}_{1}+\epsilon \mathcal{L}_{2}+\mathcal{L}_{3}, \\
3 p_{2}=\epsilon \mathcal{L}_{1}+\epsilon^{2} \mathcal{L}_{2}+\mathcal{L}_{3}, \\
3 p_{3}=\mathcal{L}_{1}+\mathcal{L}_{2}+\mathcal{L}_{3} .
\end{gathered}
$$

As we will prove below, the Lagrange resolvents are essential to determine consistent deformations of cubic equations.

\section{A. Generic case}

As it was found in, Ref. 15 for $N=3$ the operator $J_{0}$ reads

$$
J_{0}=\left(\begin{array}{ccc}
3 \partial_{x} & w \partial_{x}+w_{x} & \left(2 v+w^{2}\right) \partial_{x}+\left(2 v+w^{2}\right)_{x} \\
-2 w \partial_{x} & 2 v \partial_{x}+v_{x} & (3 u+v w) \partial_{x}+2 u_{x}+2 v w_{x} \\
-v \partial_{x} & 3 u \partial_{x}+u_{x} & u w \partial_{x}+2 u w_{x}
\end{array}\right) .
$$

Thus if $d_{1}, d_{2}$, and $d_{3}$ are the degrees in $k$ of the potential functions $w, v$, and $u$, respectively, the consistency conditions (14) are

$$
\begin{gathered}
d_{1} \leqslant 1, \quad d_{2} \leqslant d_{1}+1, \\
d_{3} \leqslant d_{2}+1, \quad d_{2} \leqslant d_{3}+1,
\end{gathered}
$$

which lead to the following 12 nontrivial choices for $\left(d_{1}, d_{2}, d_{3}\right)$ :

$$
\begin{array}{lll}
(0,0,1), & (0,1,0), \quad(0,1,1), & (0,1,2), \\
(1,0,0), & (1,0,1), \quad(1,1,0), & (1,1,1), \\
(1,1,2), & (1,2,1), \quad(1,2,2), & (1,2,3) .
\end{array}
$$

By using (23) and (24) it is straightforward to determine the Newton exponent $l_{0}$ for each of the cases (25). Thus one finds three categories

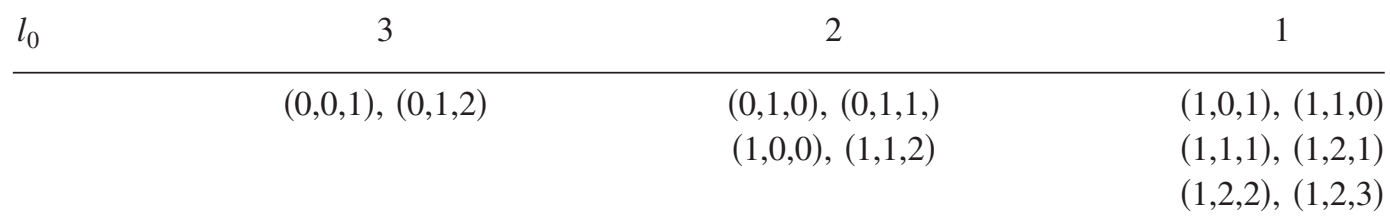

Only the cases with $l_{0}=3$ correspond to irreducible curves over the field $\mathrm{C}((k))$. We also note here that our deformations for the trigonal curves (5) in the generic case allow one to have only the curves with genus less than or equal to 1 (the details will be discussed elsewhere).

Once the Newton exponent $l_{0}$ is known, in order to derive the associated hierarchy of integrable deformations according to our scheme, two steps are still required:

(1) To determine the functions $R(z, \mathbf{p})=\sum_{i} f_{i}(z) p_{i}$ such that the components of $\nabla_{\mathbf{u}} R$ are in $\mathrm{C}((k))$ with $k=z^{l_{0}}$.

(2) To find the explicit form of the gradients $\nabla_{\mathbf{u}} R$ in terms of the potentials. 
Both problems admit a convenient treatment in terms of Lagrange resolvents. Thus by introducing the following element $\sigma_{0}$ of the Galois group of the curve:

$$
\sigma_{0}\left(p_{i}\right)(z):=p_{i}\left(\epsilon_{0} z\right), \quad \epsilon_{0}:=e^{2 \pi i / l_{0}},
$$

we see that our first problem can be fixed by determining functions $R$ invariants under $\sigma_{0}$, i.e., $R\left(\epsilon_{0} z, \sigma_{0} \mathbf{p}\right)=R(z, \mathbf{p})$. In this way, we have the following forms of $R$.

For the case $l_{0}=3$, the element $\sigma_{0}$ is given by the permutation

$$
\sigma_{0}=\left(\begin{array}{lll}
p_{1} & p_{2} & p_{3} \\
p_{2} & p_{3} & p_{1}
\end{array}\right)
$$

or, in terms of Lagrange resolvents,

$$
\sigma_{0}=\left(\begin{array}{ccc}
\mathcal{L}_{1} & \mathcal{L}_{2} & \mathcal{L}_{3} \\
\epsilon^{2} \mathcal{L}_{1} & \epsilon \mathcal{L}_{2} & \mathcal{L}_{3}
\end{array}\right) .
$$

Thus we get the invariant functions

$$
R=z f_{1}\left(z^{3}\right) \mathcal{L}_{1}+z^{2} f_{2}\left(z^{3}\right) \mathcal{L}_{2}+f_{3}\left(z^{3}\right) \mathcal{L}_{3},
$$

with $f_{i}\left(z^{3}\right)$ being arbitrary functions in $\mathrm{C}\left(\left(z^{3}\right)\right)$.

For the case $l_{0}=2, \sigma_{0}^{2}$ is the identity permutation, so that under the action of $\sigma_{0}$ two branches are interchanged while the other remains invariant. If we label the branches in such a way that

$$
\sigma_{0}=\left(\begin{array}{lll}
p_{1} & p_{2} & p_{3} \\
p_{2} & p_{1} & p_{3}
\end{array}\right)
$$

then

$$
\sigma_{0}=\left(\begin{array}{ccc}
\mathcal{L}_{1} & \mathcal{L}_{2} & \mathcal{L}_{3} \\
\mathcal{L}_{2} & \mathcal{L}_{1} & \mathcal{L}_{3}
\end{array}\right),
$$

and we obtain the invariant functions

$$
R=f_{1}\left(z^{2}\right)\left(\mathcal{L}_{1}+\mathcal{L}_{2}\right)+z f_{2}\left(z^{2}\right)\left(\mathcal{L}_{1}-\mathcal{L}_{2}\right)+f_{3}\left(z^{2}\right) \mathcal{L}_{3},
$$

where $f_{i}\left(z^{2}\right)$ are arbitrary functions in $\mathrm{C}\left(\left(z^{2}\right)\right)$.

For the case $l_{0}=1$, we have $z=k$ and $\sigma_{0}$ is the identity, so that any function $R(k, \mathbf{p})$ is invariant under $\sigma_{0}$.

Now the problem of finding the gradients of $R$ reduces to determine the gradients of the Lagrange resolvents. To this end we differentiate (23) and obtain

$$
\begin{gathered}
\mathcal{L}_{2} \nabla_{\mathbf{u}} \mathcal{L}_{1}+\mathcal{L}_{1} \nabla_{\mathbf{u}} \mathcal{L}_{2}=(2 w, 3,0)^{\top}, \\
\mathcal{L}_{1}^{2} \nabla_{\mathbf{u}} \mathcal{L}_{1}+\mathcal{L}_{2}^{2} \nabla_{\mathbf{u}} \mathcal{L}_{2}=\left(2 w^{2}+3 v, 3 w, 9\right)^{\top},
\end{gathered}
$$

so that

$$
\begin{aligned}
& \left(\mathcal{L}_{1}^{3}-\mathcal{L}_{2}^{3}\right) \nabla_{\mathbf{u}} \mathcal{L}_{1}=\left(\left(2 w^{2}+3 v\right) \mathcal{L}_{1}-2 w \mathcal{L}_{2}^{2}, 3\left(w \mathcal{L}_{1}-\mathcal{L}_{2}^{2}\right), 9 \mathcal{L}_{1}\right)^{\top} \\
& \left(\mathcal{L}_{2}^{3}-\mathcal{L}_{1}^{3}\right) \nabla_{\mathbf{u}} \mathcal{L}_{2}=\left(\left(2 w^{2}+3 v\right) \mathcal{L}_{2}-2 w \mathcal{L}_{1}^{2}, 3\left(w \mathcal{L}_{2}-\mathcal{L}_{1}^{2}\right), 9 \mathcal{L}_{2}\right)^{\top}
\end{aligned}
$$

Hence the gradients of the generic density $R$ for (29) and (32) are given as follows.

For $l_{0}=3$, we have 


$$
\nabla_{\mathbf{u}} R=\frac{z f_{1}\left(z^{3}\right)}{\mathcal{L}_{1}^{3}-\mathcal{L}_{2}^{3}}\left(\begin{array}{c}
\left(2 w^{2}+3 v\right) \mathcal{L}_{1}-2 w \mathcal{L}_{2}^{2} \\
3\left(w \mathcal{L}_{1}-\mathcal{L}_{2}^{2}\right) \\
9 \mathcal{L}_{1}
\end{array}\right)-\frac{z^{2} f_{2}\left(z^{3}\right)}{\mathcal{L}_{1}^{3}-\mathcal{L}_{2}^{3}}\left(\begin{array}{c}
\left(2 w^{2}+3 v\right) \mathcal{L}_{2}-2 w \mathcal{L}_{1}^{2} \\
3\left(w \mathcal{L}_{2}-\mathcal{L}_{1}^{2}\right) \\
9 \mathcal{L}_{2}
\end{array}\right)+f_{3}\left(z^{3}\right)\left(\begin{array}{l}
1 \\
0 \\
0
\end{array}\right)
$$

For $l_{0}=2$, we get

$$
\begin{aligned}
& \nabla_{\mathbf{u}} R=\frac{f_{1}\left(z^{2}\right)}{\mathcal{L}_{1}^{3}-\mathcal{L}_{2}^{3}}\left(\begin{array}{c}
\left(2 w^{2}+3 v\right)\left(\mathcal{L}_{1}-\mathcal{L}_{2}\right)+2 w\left(\mathcal{L}_{1}^{2}-\mathcal{L}_{2}^{2}\right) \\
3\left(w \mathcal{L}_{1}-\mathcal{L}_{2}^{2}\right)-3\left(w \mathcal{L}_{2}-\mathcal{L}_{1}^{2}\right) \\
9\left(\mathcal{L}_{1}-\mathcal{L}_{2}\right)
\end{array}\right) \\
& +\frac{z f_{2}\left(z^{2}\right)}{\mathcal{L}_{1}^{3}-\mathcal{L}_{2}^{3}}\left(\begin{array}{c}
\left(2 w^{2}+3 v\right)\left(\mathcal{L}_{1}+\mathcal{L}_{2}\right)-2 w\left(\mathcal{L}_{1}^{2}+\mathcal{L}_{2}^{2}\right) \\
3\left(w \mathcal{L}_{1}-\mathcal{L}_{2}^{2}\right)+3\left(w \mathcal{L}_{2}-\mathcal{L}_{1}^{2}\right) \\
9\left(\mathcal{L}_{1}+\mathcal{L}_{2}\right)
\end{array}\right)+f_{3}\left(z^{2}\right)\left(\begin{array}{l}
1 \\
0 \\
0
\end{array}\right)
\end{aligned}
$$

From these expressions and (28) and (31) it follows that the corresponding components of $\nabla_{\mathbf{u}} R$ are in $\mathrm{C}((k))$.

Example 1: The case $l_{0}=3$ with $\left(d_{1}, d_{2}, d_{3}\right)=(0,0,1)$. Taking into account (24) and (23) it is clear that there are two trivial equations corresponding to $w_{0}$ and $u_{1}$. Then, we take for the potentials

$$
w=1, \quad v=v_{0}(x, t), \quad u=k+u_{0}(x, t) .
$$

Thus, by using (29) with

$$
f_{1} \equiv f_{3} \equiv 0, \quad f_{2}\left(z^{3}\right)=\frac{27(1-\sqrt{3} i)}{4} z^{3},
$$

we obtain

$$
\begin{gathered}
v_{0 t}=\frac{5}{3}\left(2+27 u_{0}+9 v_{0}\right) u_{0 x}+\frac{5}{18}\left(7+54 u_{0}+36 v_{0}+27 v_{0}^{2}\right) v_{0 x}, \\
u_{0 t}=\frac{5}{18}\left(-1-54 u_{0}+27 v_{0}^{2}\right) u_{0 x}+\frac{5}{9} v_{0}\left(2+27 u_{0}+9 v_{0}\right) v_{0 x} .
\end{gathered}
$$

It can be checked that this system corresponds to the one obtained by setting $M=0, N=3$ in (19), and $\alpha=0, n=5$ in (21).

Example 2: The case $l_{0}=2$ with $\left(d_{1}, d_{2}, d_{3}\right)=(0,1,0),\left(l_{0}=2\right)$. From (24) and (23) we see that $v_{1 t}=0$. We then take

$$
w=w_{0}(x, t), \quad v=k+v_{0}(x, t), \quad u=u_{0}(x, t),
$$

and

$$
f_{1}\left(z^{2}\right)=z^{4}, \quad f_{2} \equiv f_{3} \equiv 0 .
$$

Thus it follows

$$
\begin{gathered}
w_{0 t}=4\left(w_{0} u_{0 x}+v_{0} v_{0 x}+u_{0} w_{0 x}\right), \\
v_{0 t}=-2\left(w_{0}^{2} u_{0 x}-2 u_{0} v_{0 x}+u_{0} w_{0} w_{0 x}\right)+2 v_{0}\left(2 u_{0 x}-w_{0} v_{0 x}\right), \\
u_{0 t}=-2\left(v_{0} w_{0} u_{0 x}+u_{0}\left(-2 u_{0 x}+w_{0} v_{0 x}+v_{0} w_{0 x}\right)\right) .
\end{gathered}
$$

It turns out that this system can also be found among the Whitham deformations, by setting $M$ $=1, N=2$ in (19), and $\alpha=0, n=4$ in (21). 
Example 3: The case $l_{0}=2$ with $\left(d_{1}, d_{2}, d_{3}\right)=(1,0,0)$. From (23) and (24) it is easy to see that $\left(u_{0} / w_{1}\right)_{t}=0$. If we choose

$$
\begin{gathered}
w(k, x, t)=w_{1}(x, t) k+w_{0}(x, t), \quad v(k, x, t)=v_{0}(x, t), \\
u(k, x, t)=w_{1}(x, t),
\end{gathered}
$$

and set

$$
f_{1}\left(z^{2}\right)=z^{4}, \quad f_{2} \equiv f_{3} \equiv 0
$$

in (32), then the following system arises:

$$
\begin{gathered}
w_{1 t}=2 w_{1}^{-2}\left(w_{1} w_{0 x}-w_{0} w_{1 x}\right), \\
w_{0 t}=2 w_{1}^{-3}\left(w_{1}\left(v_{0 x}+w_{0} w_{0 x}\right)-\left(2 v_{0}+w_{0}^{2}\right) w_{1 x}\right), \\
v_{0 t}=w_{1}^{-3}\left(-4 w_{1} w_{1 x}+2 v_{0}\left(w_{1} w_{0 x}-w_{0} w_{1 x}\right)\right) .
\end{gathered}
$$

This is one of the flows in the dispersionless Dym hierarchy corresponding to the curve, $w_{1} k=p$ $-w_{0}-v_{0} p^{-1}-w_{1} p^{-2}$. Also note that the linear flow, i.e., $w_{1 t}=c w_{1 x}$, etc., with $c=$ constant, can be obtained by the choice $f_{2} \propto z^{-2}$ with $f_{1}=f_{3}=0$.

Example 4: The case $l_{0}=1$ with $\left(d_{1}, d_{2}, d_{3}\right)=(1,0,1)$. From (23) and (24) one finds that $\left(u_{1} / w_{1}\right)_{t}=0$. By setting

$$
\begin{gathered}
w(k, x, t)=w_{1}(x, t) k+w_{0}(x, t), \quad v(k, x, t)=v_{0}(x, t), \\
u(k, x, t)=w_{1}(x, t) k+u_{0}(x, t),
\end{gathered}
$$

and

$$
R=\frac{2(1+\sqrt{3} i)}{\sqrt{3}} k \mathcal{L}_{1},
$$

we obtain

$$
\begin{gathered}
w_{1 t}=u_{0 x}+w_{0 x}, \\
w_{0 t}=w_{1}^{-2}\left(w_{1}\left(v_{0 x}+u_{0} w_{0 x}\right)-\left(3+v_{0}\right) w_{1 x}-w_{0}^{2} w_{1 x}+w_{0}\left(w_{1}\left(u_{0 x}+2 w_{0 x}\right)-u_{0} w_{1 x}\right)\right), \\
v_{0 t}=w_{1}^{-2}\left(w_{1}\left(2\left(2+v_{0}\right) u_{0 x}+u_{0} v_{0 x}+w_{0} v_{0 x}+2 v_{0} w_{0 x}\right)-2\left(u_{0}\left(3+v_{0}\right)-\left(1-v_{0}\right) w_{0}\right) w_{1 x}\right), \\
u_{0 t}=w_{1}^{-2}\left(-w_{0} w_{1} u_{0 x}-3 u_{0}^{2} w_{1 x}+v_{0}\left(w_{1} v_{0 x}+\left(1-v_{0}\right) w_{1 x}\right)+u_{0}\left(w_{1}\left(4 u_{0 x}+w_{0 x}\right)+w_{0} w_{1 x}\right)\right) .
\end{gathered}
$$

We also note that the linear flow is obtained by choosing $R \propto \mathcal{L}_{1}$, and the higher flows in the hierarchy can be obtained by $R \propto k^{n} \mathcal{L}_{1}$.

Example 5: The case $l_{0}=1$ with $\left(d_{1}, d_{2}, d_{3}\right)=(1,1,0)$. From (23) and (24) we deduce that $\left(v_{1} / w_{1}\right)_{t}=0$. Now we take

$$
\begin{gathered}
w(k, x, t)=w_{1}(x, t) k+w_{0}(x, t), \quad v(k, x, t)=w_{1}(x, t) k+v_{0}(x, t), \\
u(k, x, t)=u_{0}(x, t)
\end{gathered}
$$

and set 


$$
R=\frac{\sqrt{3}+i}{2 \sqrt{3}} k \mathcal{L}_{2} .
$$

Then the following system is obtained:

$$
\begin{gathered}
w_{1 t}=2 u_{0 x}-v_{0 x}, \\
w_{0 t}=w_{1}^{-2}\left(w_{1}\left(\left(3+2 w_{0}\right) u_{0 x}-\left(2+w_{0}\right) v_{0 x}+\left(2 u_{0}-v_{0}\right) w_{0 x}\right)+\left(v_{0}\left(2+w_{0}\right)-u_{0}\left(3+2 w_{0}\right)\right) w_{1 x}\right), \\
v_{0 t}=w_{1}^{-2}\left(w_{1}\left(\left(-2+4 v_{0}-2 w_{0}\right) u_{0 x}+\left(2 u_{0}-3 v_{0}+w_{0}\right) v_{0 x}\right)+\left(v_{0}\left(2 v_{0}-w_{0}\right)+u_{0}\left(3-4 v_{0}+2 w_{0}\right)\right) w_{1 x}\right), \\
u_{0 t}=w_{1}^{-2}\left(\left(-2 v_{0}+w_{0}\right) w_{1} u_{0 x}-6 u_{0}^{2} w_{1 x}+u_{0}\left(w_{1}\left(8 u_{0 x}-3 v_{0 x}+w_{0 x}\right)+2\left(2 v_{0}-w_{0}\right) w_{1 x}\right)\right) .
\end{gathered}
$$

\section{B. Hamiltonian structures}

The general structure of integrable deformations (11) does not exhibit a direct Hamiltonian form. However, the analysis of particular cases reveals the presence of certain Hamiltonian structures. We look for a Hamiltonian operator $J$ such that for certain appropriate densities $R$ it verifies

$$
J_{0}\left(T \nabla_{\mathbf{u}} R\right)_{+}=J\left(\nabla_{\mathbf{u}} R\right)_{+},
$$

where

$$
T:=\left(\begin{array}{ccc}
1 & -w & -v \\
0 & 1 & -w \\
0 & 0 & 1
\end{array}\right)
$$

Thus, if (33) holds then the flows (11) can be written in the pre-Hamiltonian form

$$
\partial_{t} \mathbf{u}=J\left(\nabla_{\mathbf{u}} R\right)_{+} .
$$

To achieve our aim we require a $k$-independent operator $T_{0}$ verifying

$$
T \nabla_{\mathbf{u}} R=T_{0} \nabla_{\mathbf{u}} R,
$$

so that $J:=J_{0} \cdot T_{0}$ is a Hamiltonian operator.

Let us consider first the case $l_{0}=3$. It involves two classes of cubic curves.

For the case with $\left(d_{1}, d_{2}, d_{3}\right)=(0,0,1)$, the potentials are of the form

$$
w=w_{0}(x), \quad v(x)=v_{0}(x), \quad u=u_{0}(x)+k u_{1}(x) .
$$

The matrix $T$ is $k$ independent so that by setting $J=J_{0} \cdot T$ we find the Hamiltonian operator

$$
J=\left(\begin{array}{ccc}
3 \partial_{x} & -2 \partial_{x} \cdot w & -\partial_{x} \cdot v \\
-2 w \partial_{x} & 2 w \partial_{x} \cdot w+2 v \partial_{x}+v_{x} & (3 u+v w) \partial_{x}+2 u_{x}+w v_{x} \\
-v \partial_{x} & (3 u+v w) \partial_{x}+v w_{x}+u_{x} & v \partial_{x} \cdot v-2 u w \partial_{x}-(u w)_{x}
\end{array}\right) .
$$

It represents the dispersionless limit of the Hamiltonian structure of the Boussinesq hierarchy.

For the case with $(0,1,2)$ the potentials now are

$$
\begin{gathered}
w=w_{0}(x), \quad v(x)=v_{0}(x)+k v_{1}(x), \\
u=u_{0}(x)+k u_{1}(x)+k^{2} u_{2}(x) .
\end{gathered}
$$

From (33) one deduces 


$$
\begin{gathered}
T \nabla_{\mathbf{u}} \mathcal{L}_{i}=T_{0} \nabla_{\mathbf{u}} \mathcal{L}_{i}, \quad i=1,2, \\
T \nabla_{\mathbf{u}} \mathcal{L}_{3}=\mathcal{L}_{3},
\end{gathered}
$$

where $T_{0}$ is the $k$-independent matrix

$$
T_{0}=\left(\begin{array}{ccc}
-2 & w & 0 \\
0 & 1 & -w \\
0 & 0 & 1
\end{array}\right)
$$

Moreover $J:=J_{0} \cdot T_{0}$ takes the Hamiltonian form

$$
J=\left(\begin{array}{ccc}
-6 \partial_{x} & 4 \partial_{x} \cdot w & 2 \partial_{x} \cdot v \\
4 w \partial_{x} & -2 w \partial_{x} \cdot w+2 v \partial_{x}+v_{x} & (3 u-v w) \partial_{x}+2 u_{x}-w v_{x} \\
2 v \partial_{x} & (3 u-v w) \partial_{x}-v w_{x}+u_{x} & -2 u w \partial_{x}-(u w)_{x}
\end{array}\right)
$$

Thus by setting

$$
R=z f_{1}\left(z^{3}\right) \mathcal{L}_{1}+z^{2} f_{2}\left(z^{3}\right) \mathcal{L}_{2},
$$

equation (11) reduces to the form (34).

For the remaining cases of $l_{0}=2$ and $l_{0}=1$, the situation is as follows:

(1) For the sets of degrees $(0,1,0)$ and $(0,1,1)$ for $l_{0}=2$, the identities $(37)$ with the same operator (38) hold, so that by setting

$$
R=f_{1}\left(z^{2}\right)\left(\mathcal{L}_{1}+\mathcal{L}_{2}\right)+z f_{2}\left(z^{2}\right)\left(\mathcal{L}_{1}-\mathcal{L}_{2}\right),
$$

Eq. (11) reduces to the form (34) with the Hamiltonian operator (39).

(2) For the sets of degrees (two cases of $l_{0}=2$ and all the cases of $l_{0}=1$ ),

$$
\begin{aligned}
& (1,0,0),(1,0,1),(1,1,0),(1,1,1), \\
& (1,1,2),(1,2,1),(1,2,2),(1,2,3),
\end{aligned}
$$

there is no $k$-independent operator $T_{0}$ satisfying (36) for $\nabla_{\mathbf{u}} \mathcal{L}_{i},(i=1,2)$.

\section{Deformations of cubic curves with $w=0$}

Deformations of cubic curves of the form

$$
p^{3}-v p-u=0
$$

cannot be obtained simply by setting $w=0$ in the above analysis. Indeed, as it is clear from the expression (24) for $J_{0}$, the constraint $w=0$ does not constitute a reduction of the flows (11). Therefore, we must apply our deformation scheme to (40) directly.

In terms of the branches $p_{i}$ the condition $w=0$ reads

$$
p_{1}+p_{2}+p_{3}=0
$$

which is preserved by deformations

$$
\partial_{t} p_{i}=\partial_{x}\left(a_{1}+a_{2} p_{i}+a_{3} p_{i}^{2}\right)
$$

satisfying 


$$
3 a_{1}=-\left(p_{1}^{2}+p_{2}^{2}+p_{3}^{2}\right) a_{3} .
$$

By expressing the potentials as functions of the branches $p_{1}$ and $p_{2}$,

$$
v=p_{1}^{2}+p_{2}^{2}+p_{1} p_{2}, \quad u=-\left(p_{1}^{2} p_{2}+p_{1} p_{2}^{2}\right),
$$

and using (41) and (42), we obtain

$$
\partial_{t} \mathbf{u}=\mathcal{J}_{0} \mathbf{a}, \quad \mathbf{u}:=\left(\begin{array}{ll}
v & u
\end{array}\right)^{\top}, \quad \mathbf{a}:=\left(a_{1} a_{2}\right)^{\top},
$$

where

$$
\mathcal{J}_{0}=\left(\begin{array}{cc}
2 p_{1}+p_{2} & 2 p_{2}+p_{1} \\
-2 p_{1} p_{2}-p_{2}^{2} & -2 p_{1} p_{2}-p_{1}^{2}
\end{array}\right) \partial_{x}\left(\begin{array}{cc}
p_{1} & \frac{1}{3} p_{1}^{2}-\frac{2}{3}\left(p_{2}^{2}+p_{1} p_{2}\right) \\
p_{2} & \frac{1}{3} p_{2}^{2}-\frac{2}{3}\left(p_{1}^{2}+p_{1} p_{2}\right)
\end{array}\right)=\left(\begin{array}{cc}
2 v \partial_{x}+v_{x} & 3 u \partial_{x}+2 u_{x} \\
3 u \partial_{x}+u_{x} & \frac{1}{3}\left(2 v^{2} \partial_{x}+2 v v_{x}\right)
\end{array}\right) .
$$

According to our strategy for finding consistent deformations, we use Lenard-type relations

$$
\mathcal{J}_{0} \mathbf{R}=0, \quad \mathbf{R}:=\left(r_{1} r_{2}\right)^{\top}, \quad r_{i} \in \mathbb{C}((k)),
$$

to generate systems of the form

$$
\mathbf{u}_{t}=\mathcal{J}_{0} \mathbf{a}, \quad \mathbf{a}:=\mathbf{R}_{+} .
$$

Here $(\cdot)_{+}$and $(\cdot)_{-}$indicate the parts of non-negative and negative powers in $k$, respectively. Now from the identity

$$
\mathcal{J}_{0} \mathbf{a}=\mathcal{J}_{0} \mathbf{R}_{+}=-\mathcal{J}_{0} \mathbf{R}_{-},
$$

it is clear that a sufficient condition for the consistency of (46) is that the degrees $d_{2}$ and $d_{3}$ of $v$ and $u$ as polynomials of $k$ satisfy

$$
d_{3} \leqslant d_{2}+1, \quad 2 d_{2} \leqslant d_{3}+1 .
$$

Hence only four nontrivial cases arise for $\left(d_{2}, d_{3}\right)$

$$
(0,1), \quad(1,1), \quad(1,2), \quad(2,3) .
$$

We notice that they represent the dispersionless versions of the standard Boussinesq hierarchy and all three hidden hierarchies found by Antonowicz, Fordy, and Liu for the third-order spectral problem. $^{21}$

Solutions of the Lenard relation can be generated by noticing that the operator $\mathcal{J}_{0}$ admits the factorization

$$
\mathcal{J}_{0}=U^{\top} \cdot \frac{1}{3}\left(\begin{array}{cc}
2 & -1 \\
-1 & 2
\end{array}\right) \partial_{x} \cdot U
$$

where

$$
U:=\left(\begin{array}{ll}
2 p_{1}+p_{2} & -2 p_{1} p_{2}-p_{2}^{2} \\
2 p_{2}+p_{1} & -2 p_{1} p_{2}-p_{1}^{2}
\end{array}\right)=\left(\begin{array}{ll}
\frac{\partial v}{\partial p_{1}} & \frac{\partial u}{\partial p_{1}} \\
\frac{\partial v}{\partial p_{2}} & \frac{\partial u}{\partial p_{2}}
\end{array}\right) .
$$

This shows two things,

(i) $\mathcal{J}_{0}$ is a Hamiltonian operator.

(ii) The gradients $\nabla_{\mathbf{u}} p_{i}$ of the branches $p_{1}$ and $p_{2}$ solve the Lenard relations. 
Thus our candidates to deformations are the equations of the form

$$
\partial_{t} \mathbf{u}=\mathcal{J}_{0}\left(\nabla_{\mathbf{u}} R\right)_{+}, \quad R(z, \mathbf{p})=f_{1}(z) p_{1}+f_{2}(z) p_{2} .
$$

At this point one applies the same strategy as that used for the curves (7) Sec. III A. We first determine the Newton exponents of the four cases (47) which turn out to be given by

\begin{tabular}{cccc}
$l_{0}$ & 2 & 1 \\
\hline & $(0,1)$ & $(1,1)$ & $(2,3)$
\end{tabular}

Then, with the help of Lagrange resolvents, we characterize the functions $R(z, \mathbf{p})$ verifying $\nabla_{\mathbf{u}} R$ $\in \mathbb{C}((k))$ with $k=z^{l_{0}}$. In summary, one finds the following.

For the case $l_{0}=3$,

$$
R=z f_{1}\left(z^{3}\right) \mathcal{L}_{1}+z^{2} f_{2}\left(z^{3}\right) \mathcal{L}_{2}, \quad k=z^{3} .
$$

For the case $l_{0}=2$,

$$
R=f_{1}\left(z^{2}\right)\left(\mathcal{L}_{1}+\mathcal{L}_{2}\right)+z f_{2}\left(z^{2}\right)\left(\mathcal{L}_{1}-\mathcal{L}_{2}\right), \quad k=z^{2} .
$$

For the case $l_{0}=1$, we have $z=k$, so that any function $R(k, \mathbf{p})=f_{1}(k) \mathcal{L}_{1}+f_{2}(k) \mathcal{L}_{2}$ is appropriate.

Example 1: The case $l_{0}=3$ with $\left(d_{2}, d_{3}\right)=(1,2)$. From (44) and (45) we have that $u_{2 t}=0$. Then if one takes

$$
u(k, x, t)=k^{2}+u_{1}(x, t) k+u_{0}(x, t), \quad v(k, x, t)=v_{1}(x, t) k+v_{0}(x, t),
$$

and sets

$$
f_{1}\left(z^{3}\right)=\frac{1}{2}(1+i \sqrt{3}) z^{3}, \quad f_{2} \equiv 0
$$

in $(51)$, one gets

$$
\begin{gathered}
v_{1 t}=-2 u_{1 x}+\frac{5}{9} v_{1}^{2} v_{1 x}, \\
v_{0 t}=\frac{1}{9}\left(-18 u_{0 x}+v_{1}^{2} v_{0 x}+4 v_{0} v_{1} v_{1 x}\right), \\
u_{1 t}=\frac{1}{9}\left(v_{1}^{2} u_{1 x}-6 v_{0} v_{1 x}-6 v_{1} v_{0 x}+6 v_{1} u_{1} v_{1 x}\right), \\
u_{0 t}=\frac{1}{9}\left(v_{1}^{2} u_{0 x}-6 v_{0} v_{0 x}+6 u_{0} v_{1} v_{1 x}\right),
\end{gathered}
$$

i.e., the dispersionless version of the coupled Boussinesq system (3.20b) in Ref. 21.

Example 2: The case $l_{0}=2$ with $\left(d_{2}, d_{3}\right)=(1,1)$. Now, one can see that $v_{1 t}=0$. By setting

$$
u(k, x, t)=u_{1}(x, t) k+u_{0}(x, t), \quad v(k, x, t)=-k+v_{0}(x, t)
$$

and

$$
f_{1}\left(z^{2}\right)=-z^{2}, \quad f_{2} \equiv 0
$$

in (52), we find the system,

$$
\begin{gathered}
v_{0 t}=-2 u_{0 x}-2 v_{0} u_{1 x}-u_{1} v_{0 x}, \\
u_{1 t}=-4 u_{1} u_{1 x}+\frac{2}{3} v_{0 x},
\end{gathered}
$$




$$
u_{0 t}=-u_{1} u_{0 x}-3 u_{0} u_{1 x}-\frac{2}{3} v_{0} v_{0 x} .
$$

This is the dispersionless version of the system (4.13) in Ref. 21.

\section{Whitham deformations of cubic curves}

There are four types of cubic curves of the form (19) given by the following equations:

$M$ 0

$$
\begin{array}{cc}
k=p^{3}+v_{1} p+v_{0} & k=p^{2}+v_{0}+\frac{v_{1}}{p-w_{1}}
\end{array} \quad k=p+\frac{v_{1,1}}{p-w_{1}}+\frac{v_{2,1}}{p-w_{2}}
$$

Note here that the Newton exponent $l_{0}$ is given by $l_{0}=3-M$. Also in Ref. 18 , two cases in $M=1$ are shown to be conformally equivalent, i.e., $p=\infty \leftrightarrow p=w_{1}$.

For $M=0$ the Whitham deformations are reductions of our flows with $w \equiv 0$. But, in general, other Whitham deformations are not of that form. To illustrate this point let us take the class with $M=1$ and $N=2$. The corresponding Newton exponent is $l_{0}=2$ and there the branches of $\mathbf{p}$ have the following asymptotic behavior as $z \rightarrow \infty$ :

$$
\begin{gathered}
p_{1}(z)=z+\mathcal{O}\left(\frac{1}{z}\right) \\
p_{2}(z)=p_{1}(-z)=-z+\mathcal{O}\left(\frac{1}{z}\right), \\
p_{3}(z)=w_{1}+\mathcal{O}\left(\frac{1}{z}\right) .
\end{gathered}
$$

Let us consider now the Whitham flows (21) associated with the puncture at $p=\infty$,

$$
\mathbf{Q}_{0, n}=\left(z^{n}\right)_{\oplus}(\mathbf{p}) .
$$

In terms of the potentials $\mathbf{u}=(w, v, u)^{\top}$ they read

$$
\partial_{t} \mathbf{u}=J_{0} \mathbf{a},
$$

where

$$
\mathbf{a}=\left(V^{-1}\left(\begin{array}{c}
\left(z^{n}\right)_{\oplus}\left(p_{1}\right) \\
\left(z^{n}\right)_{\oplus}\left(p_{2}\right) \\
\left(z^{n}\right)_{\oplus}\left(p_{3}\right)
\end{array}\right)\right)_{+} .
$$

One easily sees that all matrix elements of $V^{-1}$ are of order $\mathcal{O}(1 / z)$ with the exception of

$$
\left(V^{-1}\right)_{13}=1+\mathcal{O}\left(\frac{1}{z}\right) .
$$

On the other hand, we have

$$
\left(z^{n}\right)_{\oplus}\left(p_{1}\right)=z^{n}+\mathcal{O}\left(\frac{1}{z}\right)
$$




$$
\begin{gathered}
\left(z^{n}\right)_{\oplus}\left(p_{2}\right)=(-z)^{n}+\mathcal{O}\left(\frac{1}{z}\right), \\
\left(z^{n}\right)_{\oplus}\left(p_{3}\right)=\left(z^{n}\right)_{\oplus}\left(w_{1}\right)+\mathcal{O}\left(\frac{1}{z}\right) .
\end{gathered}
$$

Therefore one gets

$$
\mathbf{a}=\left(z^{n} V^{-1}\left(\begin{array}{c}
1 \\
(-1)^{n} \\
0
\end{array}\right)\right)_{+}+\left(z^{n}\right)_{\oplus}\left(w_{1}\right) \mathbf{e}_{3},
$$

where $\mathbf{e}_{3}=(0,0,1)^{\top}$, so that Eq. (53) becomes

$$
\partial_{t} \mathbf{u}=J_{0}\left(T \nabla_{\mathbf{u}}\left[z^{n}\left(p_{1}+(-1)^{n} p_{2}\right)\right]\right)_{+}+J_{0}\left(\left(z^{n}\right)_{\oplus}\left(w_{1}\right) \mathbf{e}_{3}\right) .
$$

Similar expressions can be obtained for the deformations generated by the Whitham flows (21) for $\alpha=1$ and $n \geqslant 1$.

\section{ACKNOWLEDGMENTS}

One of the authors (L.M.A.) wishes to thank the members of the Physics Department of Lecce University for their warm hospitality. This work is partially supported by DGCYT Project BFM 2002-01607 and by the grant COFIN 2004 "Sintes:" One of the authors (Y.K.) is partially supported by NSF Grant No. DMS 0404931

${ }^{1}$ S. P. Novikov, S. V. Manakov, L. P. Pitaevski, and V. E. Zakharov, Theory of Solitons. The Inverse Scattering Method (Plenum, New York, 1984).

${ }^{2}$ E. D. Belokolos, A. I. Bobenko, V. Z. Enolski, A. R. Its, and V. B. Matveev, Algebro-Geometric Approach to Nonlinear Integrable Equations (Springer-Verlag, Berlin, 1994).

${ }^{3}$ B. Dubrovin and S. Novikov, Russ. Math. Surveys 44, 35 (1989).

${ }^{4}$ H. Flaschka, M. G. Forest, and D. W. Mclauglin, Commun. Pure Appl. Math. 33, 739 (1980).

${ }^{5}$ B. A. Dubrovin, Commun. Math. Phys. 145, 415 (1992).

${ }^{6}$ I. M. Krichever, Funct. Anal. Appl. 22, 206 (1988).

${ }^{7}$ I. M. Krichever, Commun. Pure Appl. Math. 47, 437 (1994).

${ }^{8}$ A. Aoyama and Y. Kodama, Commun. Math. Phys. 182, 185 (1996).

${ }^{9}$ I. Krichever, M. Mineev-Weinstein, P. Wiegmann, and A. Zabrodin, Physica D 198, 1 (2004).

${ }^{10}$ A. Zabrodin, Theor. Math. Phys. 142, 166 (2005).

${ }^{11}$ R. Teodorescu, E. Bettelheim, O. Agam, A. Zabrodin, and P. Wiegmann, Nucl. Phys. B 704, 407 (2005).

${ }^{12}$ R. Teodorescu, A. Zabrodin, and P. Wiegmann, Phys. Rev. Lett. 95, 044502 (2005).

${ }^{13}$ M. Manas, L. Martinez Alonso, and E. Medina, J. Phys. A 40, 4815 (1997).

${ }^{14}$ Y. Kodama and B. G. Konopelchenko, J. Phys. A 35, L489 (2002); in Deformations of Plane Algebraic Curves and Integrable Systems of Hydrodynamic type in Nonlinear Physics: Theory and Experiment II, edited by M. J. Ablowitz et al. (World Scientific, Singapore, 2003).

${ }^{15}$ B. G. Konopelchenko and L. Martínez Alonso, J. Phys. A 37, 7859 (2004).

${ }^{16}$ B. L. van der Waerden, Algebra, Vol. I (Springer-Verlag, Berlin, 1991).

${ }^{17}$ L. Redei, Introduction to Algebra (Pergamon, Oxford, 1967), Vol. I.

${ }^{18}$ I. G. Macdonald, Symmetric Functions and Hall Polynomials (Clarendon, Oxford, 1979).

${ }^{19}$ R. Y. Walker, Algebraic Curves (Springer-Verlag, Berlin, 1978).

${ }^{20}$ S. S. Abhyankar, Algebraic Geometry for Scientists and Engineers, Mathematical Surveys and Monographs Vol. 35 (American Mathematical Society, Providence, RI, 1990).

${ }^{21}$ M. Antonowicz, A. P. Fordy, and Q. P. Liu, Nonlinearity 4, 669 (1991). 